\title{
Electron streams formation and secondary two stream instability onset in the post-saturation regime of the classical Weibel instability
}

\author{
M. E. Innocenti, ${ }^{1}$ M. Lazar, ${ }^{1,2}$ S. Markidis, ${ }^{1}$ G. Lapenta, ${ }^{1}$ and S. Poedts ${ }^{1}$ \\ ${ }^{1}$ Centrum voor Plasma-Astrofysica, Departement Wiskunde, Katholieke Universiteit Leuven, \\ Celestijnenlaan 200B, B-3001 Leuven, Belgium \\ ${ }^{2}$ Institut für Theoretische Physik, Lehrstuhl IV: Weltraum- und Astrophysik, Ruhr-Universität Bochum, \\ D-44780 Bochum, Germany
}

(Received 12 March 2011; accepted 8 April 2011; published online 16 May 2011)

\begin{abstract}
The electrostatic activity in the post-saturation regime of the velocity anisotropy driven Weibel instability is investigated by means of 1D $3 \mathrm{~V}$ particle in cell simulations. Two different initial simulation configurations have been chosen to characterize the electrostatic activity in the post-saturation stage. A secondary two stream instability arises in both cases. However, significant differences occur in the thickness of the electron streams, in their initial locations, and in their effects on the bulk electron phase space distribution. An Hamiltonian description of particle motion in a 1D setting explains these differences in terms of the effective potential experienced by particles as a function of their initial perpendicular velocity. The different roles of the longitudinal electric field and the Lorentz force in the formation of electron streams are discussed. (C) 2011 American Institute of Physics. [doi:10.1063/1.3587089]
\end{abstract}

\section{INTRODUCTION}

The generation of a magnetic field due to either the classical Weibel instability ${ }^{1,2}$ or the electromagnetic current filamentation instability ${ }^{3,4}$ is a well known plasma effect, which has been investigated in a variety of scenarios. Despite the fact that in the first case, the free energy source for the instability is provided by the thermal spread anisotropy of the electron distribution, while in the second case by the presence of two counter-streaming electron beams (such a configuration is unstable also for the two stream instability, see Lazar et al. ${ }^{5}$ for a comparison of the respective growth rates and $\mathrm{Bret}^{6}$ for a hierarchy in terms of growth rate of the many linear instabilities competing in astrophysical settings), the process of generation of the magnetic field is qualitatively the same. Infinitesimal magnetic field fluctuations generate a Lorentz force $\mathbf{F}_{L}=q \mathbf{v} \times \mathbf{B}$ that causes electron separation in different regions of space. A net current in the direction of the higher velocities (classical Weibel instability) or in the streaming direction (filamentation instability) and a consequent magnetic field on the perpendicular plane are generated. An electric field grows consistently with Ampere's law.

Many authors have focused on the saturation process of the Weibel instability (e.g., Kato $^{7}$ and Califano et al. ${ }^{8}$ ) and, in particular, on the value and persistence in time of the magnetic field, especially in connection with the problem of the generation of magnetic fields in cosmological settings (see Medvedev and Loeb ${ }^{9}$, Califano, Cecchi, and Chiuderi, ${ }^{10}$ Schlickeiser and Shukla, ${ }^{11}$ Fonseca et al. ${ }^{12}$ and references therein). On the other hand, the study of the electrostatic activity after saturation has drawn less attention.

Yet, this problem is considered relevant and worth studying for two reasons. First, the post-saturation stage of the Weibel instability can explain the origin of electric field traces and electron holes that have been observed in a variety of space environments where a temperature anisotropy is expected to be present (see Briand ${ }^{13}$ and Matsumoto et al. ${ }^{14}$ and references therein). Second, the fact that an electromagnetic instability causes electrostatic turbulence can help to pinpoint one of the microscopic sources of the high anomalous transport coefficients often needed to explain observed macroscopic processes. ${ }^{15}$

The electrostatic post-saturation evolution of the Weibel instability has been previously addressed in Stockem, Dieckmann, and Schlickeiser ${ }^{16}$ and Palodhi, Califano, and Pegoraro. ${ }^{17}$ In Stockem, Dieckmann, and Schlickeiser, ${ }^{16}$ the formation of bipolar traces superimposed to the longitudinal electric field in a 1D 3V particle in cell simulation of the saturation and post-saturation stage of the velocity anisotropy driven Weibel instability is noticed but not further investigated. Palodhi, Califano, and Pegoraro, ${ }^{17}$ instead, give a more extensive explanation of the formation of electrostatic coherent structures in a $1 \mathrm{D} 3 \mathrm{~V}$ Vlasov simulation. These structures are there related to the excitation of Langmuir waves and to wave-particle resonating interaction.

Here, that analysis is extended with a detailed description of the particle dynamics originating the longitudinal electric field structures. This paper is organized as follows. In Sec. II, the dependence of the post-saturation evolution of the velocity anisotropy driven Weibel instability on the geometrical set up of the simulation is, for the first time to the authors' knowledge, precisely characterized. In Sec. III, differences in the post-saturation evolution of the two presented 1D $3 \mathrm{~V}$ simulations are addressed through the analysis of the longitudinal electric field and of phase space plots. In both cases, a secondary two stream instability which leads to the formation of electron holes ${ }^{18,19}$ in phase space arises after saturation. The dimensions and formation mechanisms of the streams appear, however, to be greatly different in the two cases. The origin of these differences can be explained by the application of the Hamiltonian description of particle 
motion, ${ }^{20}$ reviewed in Sec. IV, to the presented 1D setting. Such an approach has already been used in connection with the Weibel instability problem in Morse and Nielson ${ }^{21}$ and Yang, Arons, and Langdon. ${ }^{22}$ It is, however, applied here for the first time to the electrostatic post-saturation evolution of the instability. In Sec. V, first, the results of Morse and Nielson $^{21}$ regarding the saturation of the instability through magnetic trapping ${ }^{23}$ are extended, and then new insights are obtained as regards the origin of the streams. Finally, in Sec. VI, a parametric study is carried out to characterize how the above presented effects vary with a decreasing velocity anisotropy. Sec. VII presents a summary of the obtained results.

\section{THE WEIBEL INSTABILITY AND THE SIMULATION PARAMETERS}

The classical Weibel instability ${ }^{1}$ is driven by an anisotropy in the thermal velocities of electrons. With a bi-Maxwellian distribution function defined as

$$
f\left(v_{\|}, v_{\perp}\right)=\frac{1}{(2 \pi)^{3 / 2} v_{t h \perp} v_{t h \|}^{2}} \exp \left[-\frac{v_{\perp}^{2}}{2 v_{t h \perp}^{2}}-\frac{v_{\|}^{2}}{2 v_{t h \|}^{2}}-\frac{v_{\|}^{2}}{2 v_{t h \|}^{2}}\right],
$$

the dispersion relation reads

$$
\frac{k^{2}}{\omega_{p e}^{2}}-\frac{\omega^{2}}{\omega_{p e}^{2}}=-1+\left(A_{e}+1\right)\left[1+\frac{\omega}{\sqrt{2} v_{t h \|} k} Z\left(\frac{\omega}{\sqrt{2} v_{t h \|} k}\right)\right],
$$

where $c=1$ is used as normalization for the velocities, $\omega_{p e}$ is the plasma frequency for electrons, $Z(\zeta)$ is the plasma dispersion function, ${ }^{24}$ and $A_{e}=v_{t h \perp}^{2} / v_{t h \|}^{2}-1$ is the measure of the velocity anisotropy.

When the perpendicular thermal velocity is higher than the parallel thermal velocity $\left(A_{e}>0\right)$, a purely growing electromagnetic instability develops in the parallel colder direction, following the dispersion relation given in Eq. (2).

A plasma with $v_{t h \perp} / c=v_{t h, y} / c=0.0624$ and $v_{t h \|} / c=$ $v_{t h, x} / c=v_{t h, z} / c=0.0104$ is simulated here. Since $A_{e}=35$, the linear theory gives a continuous range of unstable wavenumbers spanning the interval $0<k d_{e}<k_{\text {cutoff }} d_{e}$, where $k_{\text {cutoff }} d_{e}=\sqrt{A_{e}}{ }^{21}$ and $d_{e}=c / \omega_{p e}$ is the electron skin depth.

Even if all the wavenumbers in this range are unstable in nature, the only unstable modes in the simulated problem are the ones supported by the box according to the formula $L_{x}=n 2 \pi / k$. This means that a wavelength must be contained inside the box an entire number $n$ of times to be able to resonate inside it. As a consequence, the combined choice of the velocity anisotropy and box dimensions can deeply affect the evolution of a simulation, at least in the nonlinear stage.

Two 1D 3V particle in cell simulations are performed with a box length equal to $L_{x} / d_{e}=10.81$ (this case is indicated from now on as "first simulation") and $L_{x} / d_{e}=8.6$. In the first simulation, the most unstable wavenumber $k_{\max }$ $d_{e}=1.74$ from the dispersion relation is allowed to resonate inside the simulation box with $n=3$ : since all other unstable wavenumbers have a growth rate $\gamma / \omega_{p e}$ which is consider-
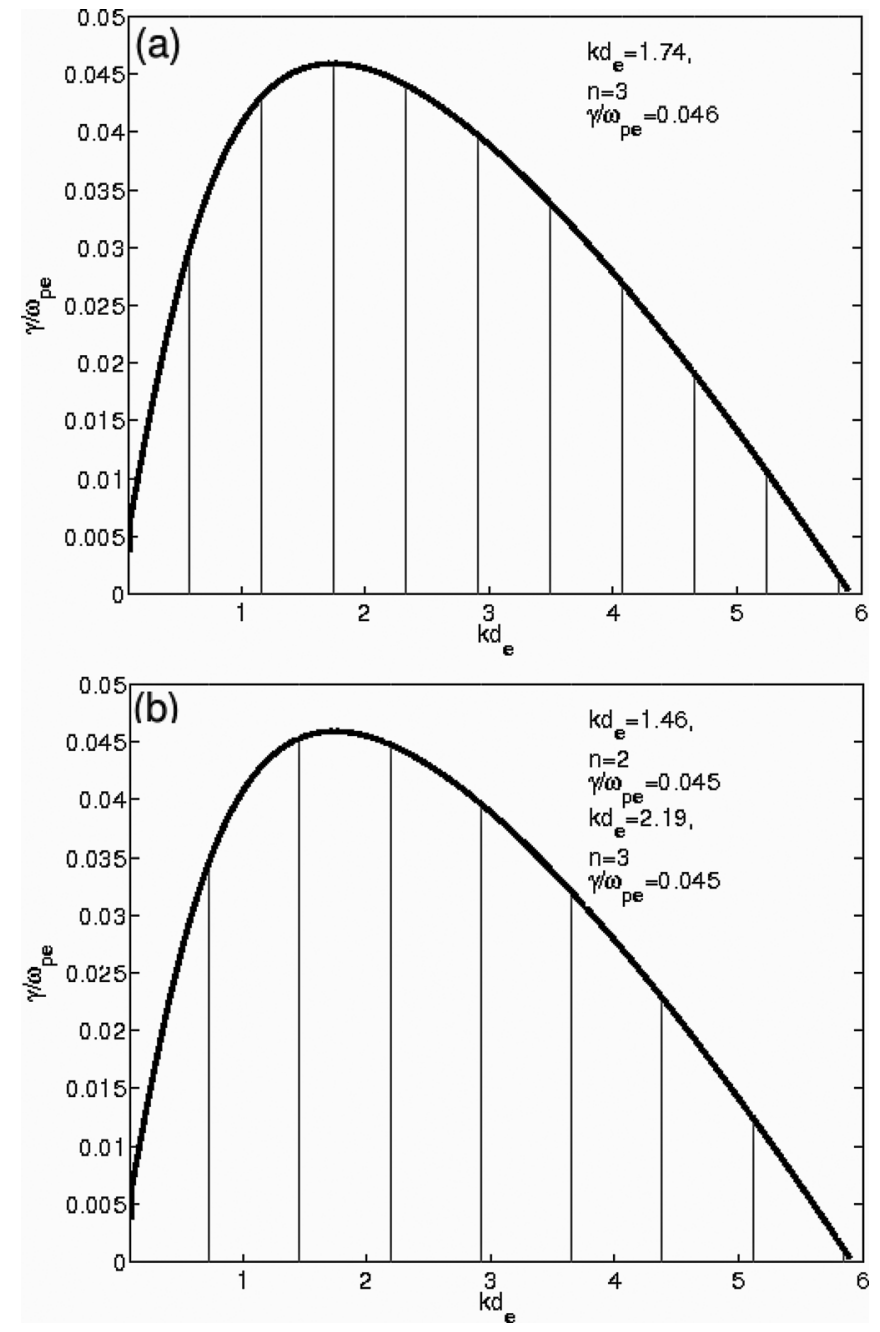

FIG. 1. Dispersion relation for the Weibel instability with $v_{t h \perp} / c=0.0624$ and $v_{t h \|} / c=0.0104$ and, superimposed as vertical lines, the unstable wavenumbers for simulations with (a) $L_{x} / d_{e}=10.81$ and (b) $L_{x} / d_{e}=8.6$.

ably lower than the one of $k_{\max } d_{e}$, no other unstable mode will presumably become dominant, also after the saturation of $k_{\max } d_{e}=1.74$. This situation is shown in Fig. 1(a), where the dispersion relation for the Weibel instability with the above mentioned perpendicular and parallel velocities is shown together with the unstable modes supported by the simulation box represented by the vertical lines. Instead, when $L_{x} / d_{e}=8.6$ (see Fig. 1(b)), the two allowed modes closer to $k_{\max } d_{e}$, the one with $n=2, k d_{e}=1.46$, and $n=3$, $k d_{e}=2.19$, have very similar growth rates according to the linear theory. Since the stabilization of unstable wavenumbers proceeds from higher to lower $k$, a likely evolution scenario in this case is first the growth as dominant mode and then the saturation of the mode with $k d_{e}=2.19$, followed by the evolution as dominant mode of $k d_{e}=1.46$.

While the first geometrical setup is the one generally adopted in simulations to allow the instability to reach its maximum theoretical growth rate, the second case is rarely simulated. It should not be regarded as forcing the simulation to evolve in an unnatural direction but as a way to reproduce in a controlled environment the progressive stabilization of wavenumbers, from the higher to the lower, as a 
(a)

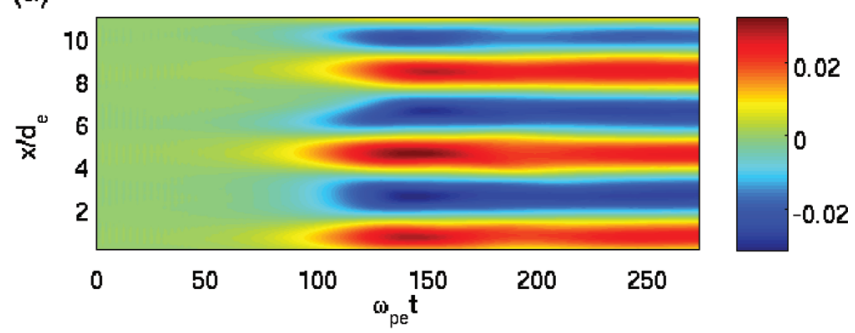

(b)

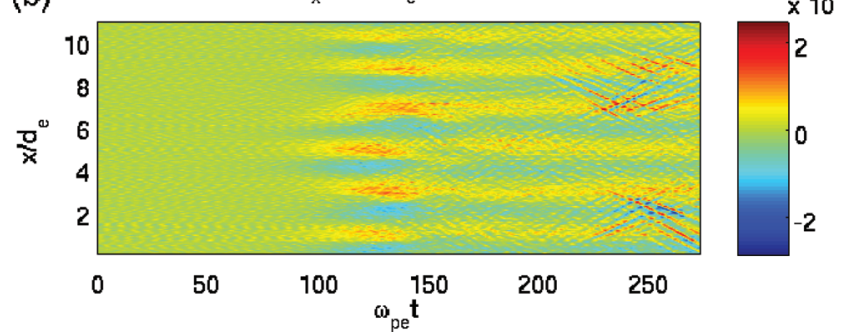

FIG. 2. (Color online) Time space plot of $B_{z}$ (panel a) and $E_{x}$ (panel b) in the simulation with $L_{x} / d_{e}=10.81$.

consequence of the progressive isotropization of the electron temperatures. $^{23}$ Notice finally that, as also, for example, in Stockem, Dieckmann, and Schlickeiser ${ }^{16}$ and in Palodhi, Califano, and Pegoraro, ${ }^{17}$ the box dimensions are chosen to have a limited number of unstable modes for the sake of clarity of exposition. Control runs with larger simulation boxes have shown behaviors qualitatively similar to the ones here presented also in presence of a higher number of unstable modes.

The simulations are performed in a $1 \mathrm{D} 3 \mathrm{~V}$ setting with iPIC3D, ${ }^{25}$ an implicit moment method parallel 3D 3V particle in cell code. One thousand and twenty-four grid cells in the $x$ direction, 1000 electrons and 1000 ions per cell, an electron to ion mass ratio equal to 1836 , and a simulation time step of $\omega_{p e} \Delta t=0.0039$ are used in both cases. Ions are loaded with the same temperature and same anisotropy as the electrons. Periodic boundary conditions are applied both to fields and particles, and an initial perturbation with $n=3$ is imposed on the magnetic field in the $z$ direction.

\section{ONSET OF THE SECONDARY TWO STREAM INSTABILITY}

The evolution of the $B_{z}$ (panel a) and $E_{x}$ (panel b) field components is shown in Figs. 2 and 3 for the simulations with $L_{x} / d_{e}=10.81$ and $L_{x} / d_{e}=8.6$, respectively. The qualitative pre-saturation evolution is similar in the two cases: the field $B_{z}$ is determined by the current arising in the direction $y$ of higher electron velocity and in turn originates, through magnetic pressure, ${ }^{16}$ an electric field in the simulated direction $x$ with a double wavenumber.

The influence of the simulation box dimension is evident in the profile of $B_{z}$ after the saturation of the mode with $n=3$ ( $\omega_{p e} t \approx 145$ in both cases), which is initially dominant in both simulations. While in Fig. 2(a) the wavenumber with $n=3$ keeps being dominant, in Fig. 3(a) the mode shift of the dominant mode from $n=3$ to $n=2$ is evident. A spectral (a)

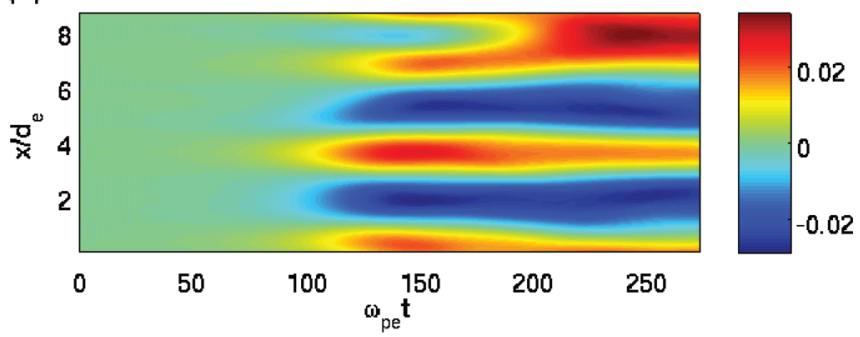

(b)

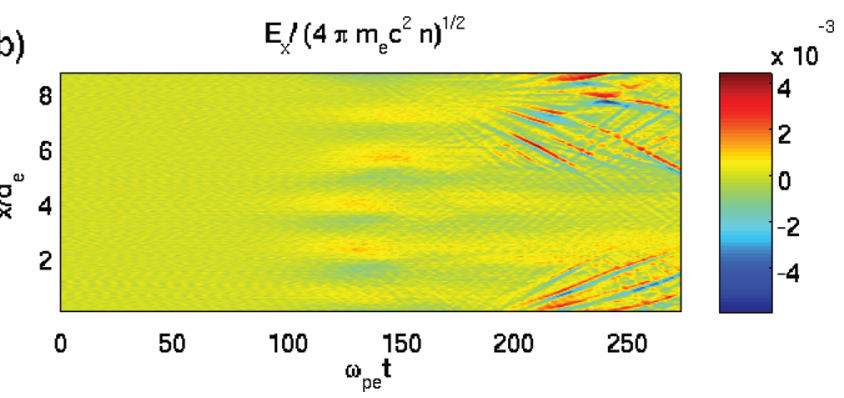

FIG. 3. (Color online) Time space plot of $B_{z}$ (panel a) and $E_{x}$ (panel b) in the simulation with $L_{x} / d_{e}=8.6$.

analysis of $B_{z}$ shows that the saturation of the mode with $n=3$ occurs at $\omega_{p e} t \approx 145$, while at $\omega_{p e} t \approx 167$ the mode with $n=2$ becomes dominant in the simulation with $L_{x}$ l $d_{e}=8.6$.

The differences in $E_{x}$ (see Figs. 2(b) and 3(b)) are more evident and reveal a different behavior after saturation in the two cases. Even if bipolar traces are superimposed in both cases to the sinusoidal-like profile of the electric field after $\omega_{p e} t \gtrsim 200$ and $\omega_{p e} t \gtrsim 180$, they appear to be different both in the spatial location of origin and in their strength. In fact, they originate approximately at the same time from multiple locations in Fig. 2(b), while a main point of origin is observable at $\omega_{p e} t \approx 180$ in Fig. 3(b). At later times $\left(\omega_{p e} t \gtrsim 200\right)$, some further weaker activity is visible. Moreover, the maximum intensity of the traces is different in the two cases: in the first simulation, the ratio between the maximum of the absolute value of $E_{x}$ after and before the formation of the streams is $r 1=\max \left(\left|E_{x, \omega_{p e} t \geq 200}\right| / \max \left(\left|E_{x, \omega_{p e} t \leq 200}\right|\right)\right.$ $=1.93$, while it increases to $r 2=\max \left(\left|E_{x, \omega_{p e} t \geq 180}\right| / \max \right.$ $\left(\left|E_{x, \omega_{p e} t \leq 180}\right|\right)=3.7$ for the second simulation. The maximum values for the electric field before the stream formation are achieved at saturation.

Figures 4 and 5 show the phase space $v_{x} / c$ vs $x / d_{e}$ in logarithmic units for the two simulations, and prove that the secondary electrostatic activity is due to a secondary two stream instability in both cases. In both figures, in panel (a), the electron streams are shown just after their formation $\left(\omega_{p e} t \approx 215\right.$ in the first simulation, $\omega_{p e} t \approx 185$ in the second), while in panel (b) the secondary two stream instability is fully developed and electron holes are visible $\left(\omega_{\text {pe }} t \approx 244\right.$ in the first simulation, $\omega_{p e} t \approx 215$ in the second).

The streams are very thin when compared to the bulk electron distribution, and the resulting electron holes develop only at the edges of it in Fig. 4. Instead, the streams are thicker and the electron holes eventually disrupt the phase space distribution in Fig. 5, which refers to the simulation 

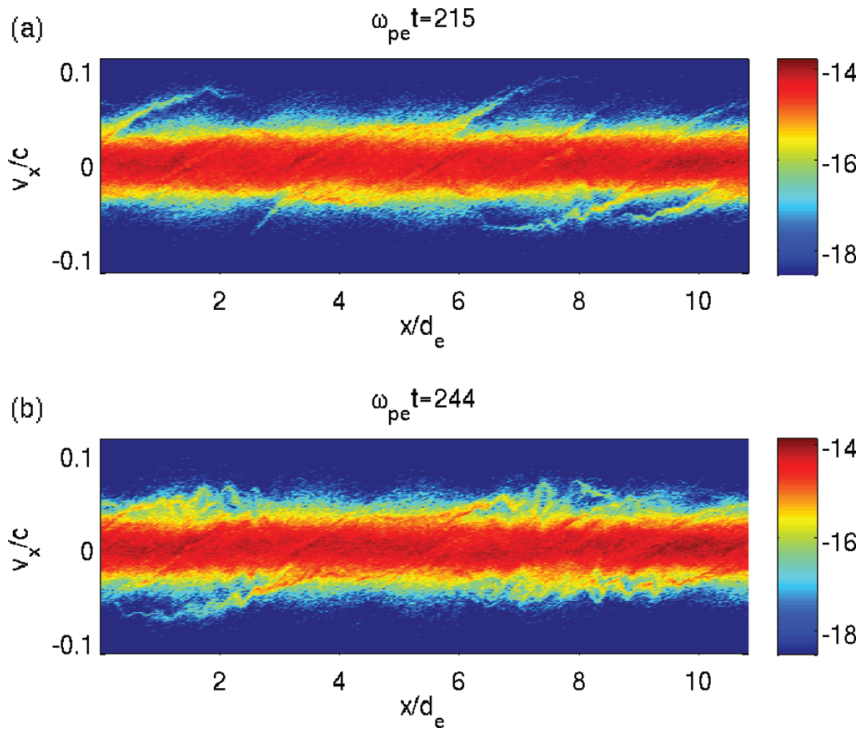

FIG. 4. (Color online) Logarithmic phase space $v_{x} / c$ vs. $x / d_{e}$ at (a) $\omega_{p e} t \approx 215$ and (b) $\omega_{p e} t \approx 244$ for the simulation without mode shift, $L_{x} /$ $d_{e}=10.81$.

with $L_{x} / d_{e}=8.6$ and shift of the dominant mode from $n=3$ to $n=2$ after saturation. Moreover, Fig. 4(a) shows multiple streams departing from multiple locations $\left(x / d_{e} \approx 3.5\right.$, $x / d_{e} \approx 5.4, x / d_{e} \approx 7.4, x / d_{e} \approx 9.2$, and $x / d_{e} \approx 10.6$, which, as more detailed later, correspond to the zeros of the magnetic field) as already noted in the plots for $E_{x}$, while only two counter-propagating streams (note that because of the periodic conditions, the forward propagating stream is reinjected at the left boundary) depart from $x / d_{e} \approx 8$ in Fig. 5(a).

\section{HAMILTONIAN DESCRIPTION OF PARTICLE MOTION IN 1D}

In the Hamiltonian formalism (see $\mathrm{Schmidt}^{20}$ for a review), the motion of particles is described in terms of the
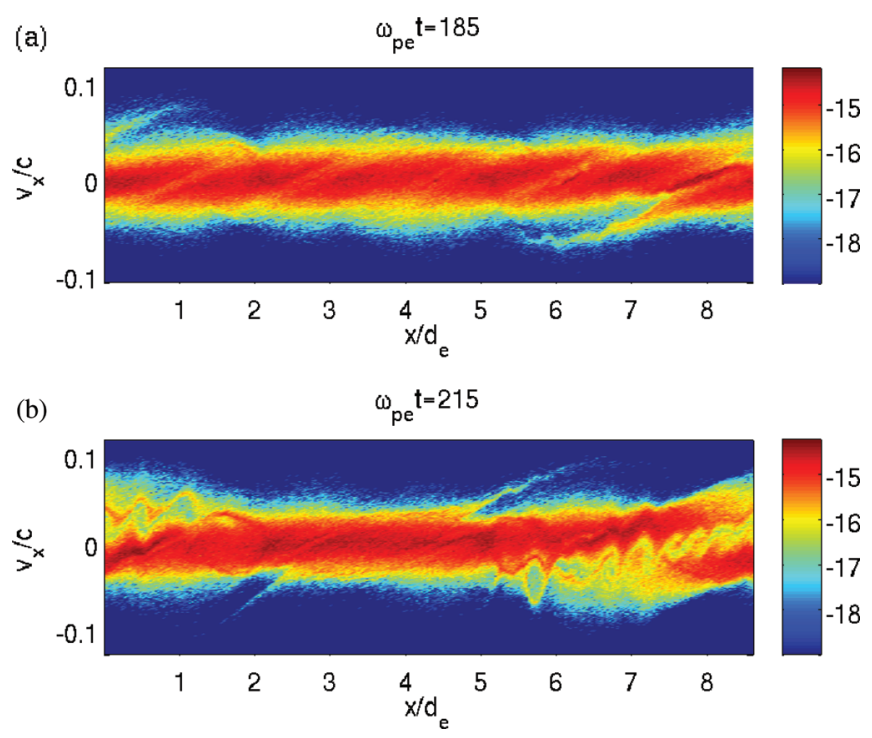

FIG. 5. (Color online) Logarithmic phase space $v_{x} / c$ vs. $x / d_{e}$ at (a) $\omega_{\text {pe }} t \approx 185$ and (b) $\omega_{\text {pe }} t \approx 215$ for the simulation with mode shift, $L_{x} l$ $d_{e}=8.6$.
Hamiltonian $H$ and of canonical coordinates and momenta $Q$ and $P$ through the following equations:

$$
\begin{gathered}
\dot{Q}_{i}=\frac{\partial H}{\partial P_{i}}, \\
\dot{P}_{i}=-\frac{\partial H}{\partial Q_{i}},
\end{gathered}
$$

where the index $i$ spans the spatial dimensions. In the considered 1D setting, where both $P_{y}$ and $P_{z}$ are constants of the motion since differentials are suppressed in the $y$ and $z$ direction, the Hamiltonian reduces to

$$
H=\frac{P_{x}^{2}}{2 m}+\Psi
$$

where

$$
\Psi=\frac{\left(P_{y}-q A_{y}\right)^{2}}{2 m}+\frac{\left(P_{z}-q A_{z}\right)^{2}}{2 m}+q V
$$

is the effective potential field, $V$ is the electric potential and $\boldsymbol{A}$ is the potential vector. Furthermore, since the Weibel instability generates a current in the direction of higher velocities, which is here $y$, and given Ampere's law with $\partial / \partial z=0$, the growth of the magnetic field is expected only in the $z$ direction, which gives $A_{y}$ as the only surviving component of the potential vector.

With

$$
P_{i}=m \dot{Q}_{i}+q A_{i}
$$

the constants of the motion $P_{z}$ and $P_{y}$ reduce, respectively, to

$$
P_{z}=m v_{z}=m v_{z 0}
$$

and

$$
P_{y}=m v_{y}+q A_{y}=m v_{y 0},
$$

since $A_{y}=0$ before $B_{z}$ is grown by the Weibel instability and $v_{z}$ is not affected by the development of the instability.

The potential field $\Psi$ thus becomes

$$
\Psi=\frac{\left(m v_{y 0}-q A_{y}\right)^{2}}{2 m}+\frac{m v_{z 0}^{2}}{2}+q V(x)=\Psi^{\star}+q V(x),
$$

where $V(x)$ is the electric potential corresponding to an electric field $\mathbf{E}\left(E_{x}, 0,0\right)$. In Morse and Nielson ${ }^{21}$ the electrostatic contribution $q V(x)$ to the effective potential is neglected, thus reducing $\Psi$ to $\Psi^{\star}$. Here, instead, the electric potential $V(x)$ stemming from $E_{x}$ is taken into consideration because it contributes to a dramatic change in the profile of the potential field for particles with $\left|v_{y 0}\right| / c \approx 0$. Equation (10) thus enables to identify the position of the potential wells for all electrons once their initial $v_{y} / c$ is known. In particular, it is possible to divide them into classes according to their initial perpendicular velocity (here, classes with $\Delta v_{y 0} / c=0.06$ for both simulations) and to follow the evolution of the effective potential field for each class during the simulations. This approach not only allows one to recover the well-known 
results of Morse and Nielson ${ }^{21}$ with regards to the magnetic trapping ${ }^{23}$ but also to go beyond and understand the origin of the secondary two stream instability, which develops after the saturation of the Weibel instability.

\section{APPLICATION OF THE HAMILTONIAN ANALYSIS TO THE SIMULATIONS}

Equation (10) allows one to calculate the effective potential field for particles once their initial velocities and the magnetic and electric fields are known. It is therefore here used to examine the behavior of the different classes of particles, sorted according to their initial perpendicular velocity $v_{y 0}$. According to Eq. (10), particles with similar initial $v_{y 0}$ will tend to group together at the minima of the potential field, thus saturating the Weibel instability through magnetic trapping. In fact, the study of the phase space divided by classes of particles for the two simulations shows that the grouping of particles occurs according to their initial perpendicular velocity, and that the streams are originated by different classes of particles in the first and second simulation also. When $L_{x} / d_{e}=10.81$, the streams are mostly due to particles with initial low $\left|v_{y}\right| / c$, while for $L_{x} / d_{e}=8.6$ the particles which are accelerated into streams are the ones with higher $\left|v_{y 0}\right| / c$. "Low" and "high" are here intended with regards to the maximum $\left|v_{y 0}\right| / c$. Moreover, it now becomes clear why in the first case the streams are originated in many locations, while in the second the thicker streams depart from just one point and why the role of the longitudinal electric field is fundamental in generating the electron streams if mode shift does not occur.

The origin of the streams in the first simulation can be understood by analyzing Figs. 6 and 7. These plots show the logarithmic phase space (panel a) and the potential field (panel b) for the particles with $-0.009 \leq v_{y 0} / c \leq 0.052$ in the simulation with $L_{x} / d_{e}=10.81$ at saturation $\left(\omega_{p e} t \approx 146\right)$ and at the time of stream formation $\left(\omega_{p e} t \approx 215\right)$, respectively. In panels (b), both the potential field without $\left(\Psi^{\star}\right)$ and with $(\Psi)$ the potential contribution $V(x)$ are shown for the particles with highest and lowest initial perpendicular velocity in the class $\left(v_{y 0}=0.052\right.$ and $\left.v_{y 0}=-0.009\right)$ to reveal the role of the electric field in determining the trapping position of electrons with $v_{y 0} / c \approx 0$. It is clear from Figs. 6 and 7 that the profile of the potential field changes considerably with the inclusion of the electrostatic contribution, especially for the particles with lower $\left|v_{y 0}\right| / c$. In fact, the electrostatic contribution is needed to recover the trapping pattern particles exhibit in phase space plots in the profile for the potential field. Note that the potential wells for particles with $v_{y 0} /$ $c=0.052$ are located at the zeros of $B_{z}\left(B_{z}\right.$ and $A_{y}$ at $\omega_{\text {pe }} t \approx 146$ are shown in Fig. 8), which correspond to the minima of $A_{y}$, since the perpendicular velocity of almost all particles in the class is positive. Instead, particles with a negative high $v_{y 0} / c$ are trapped at the maxima of $A_{y}{ }^{21}$

The role of the electrostatic electric field $E_{x}$ in freeing the particles from the potential wells can be understood by comparing Figs. 6-8. In Fig. 8, the relevant component $B_{z}$ and $A_{y}$ of the magnetic field and the vector potential are shown together with $J_{e y} B_{z} /\left(e n_{e}\right)$, where $J_{e}$ is the electron
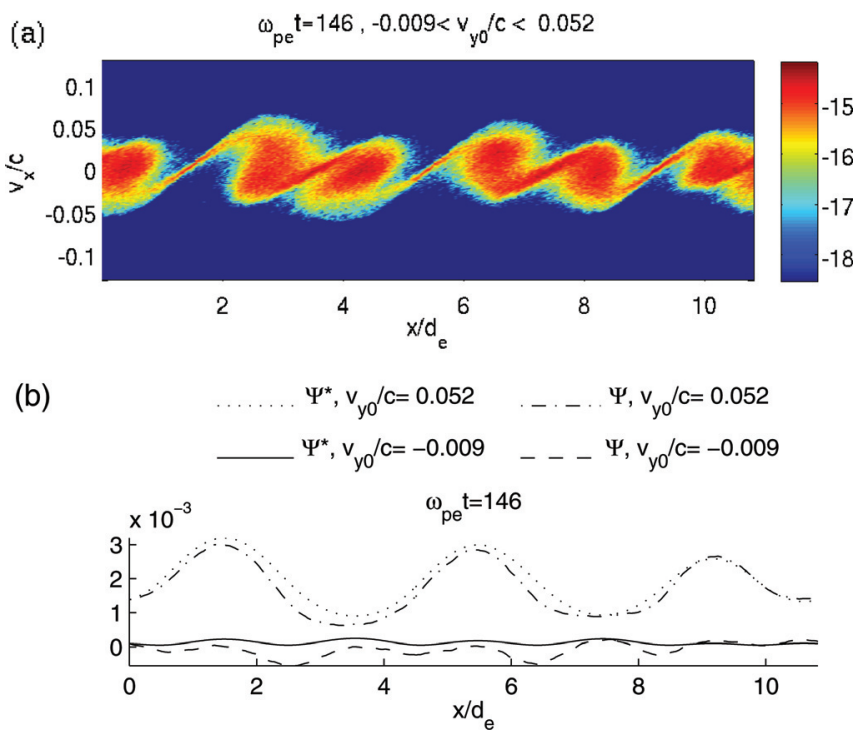

FIG. 6. (Color online) Logarithmic phase space (a) and potential fields (b) for particles with $-0.009 \leq v_{y 0} / c \leq 0.052$ at $\omega_{p e} t \approx 146$, when saturation occurs, for the simulation with $L_{x} / d_{e}=10.81$. In panel (b), the potential fields with $(\Psi)$ and without $\left(\Psi^{\star}\right)$ the electrostatic contribution are depicted for the particles of the class with extreme velocities.

current, $n_{e}$ is the electron density and $e$ is the absolute value of the normalized electric charge at $\omega_{\text {pe }} t \approx 146$, when saturation occurs. The profiles and the values of the fields are slightly modified at later times, but the zeros remain approximately unchanged. $J_{e y} B_{z} /\left(e n_{e}\right)$ is plotted instead of $E_{x}$ because it is affected by less numerical noise.

Focus, for example, on the formation of the forward propagating stream which departs from $x / d_{e} \approx 9.2$ and of the backward propagating stream departing from $x / d_{e} \approx 5.4$ in Fig. 7. The electrons which originate them are trapped at saturation time between $5.9 \lesssim x / d_{e} \lesssim 8.7$. The Lorentz force in the $x$ direction, $F_{L, x}=q v_{y} B_{z}$, tends to keep particles

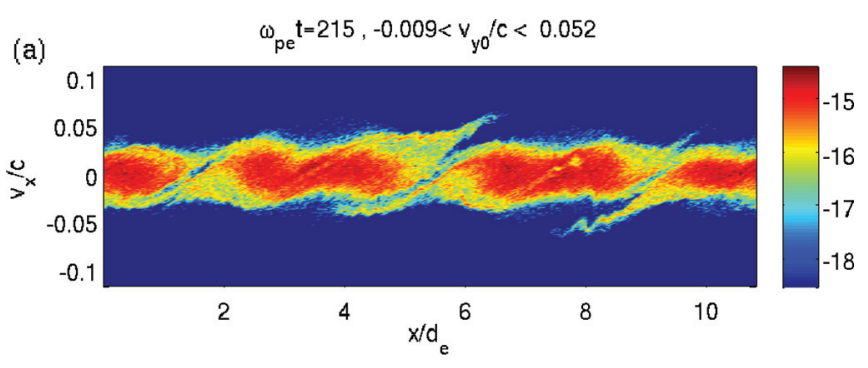

(b)

$$
\begin{aligned}
& \Psi^{*}, v_{\mathrm{y} 0} / \mathrm{c}=0.052 \ldots \Psi, \mathrm{v}_{\mathrm{yo}} / \mathrm{c}=0.052 \\
& \Psi^{\star}, \mathrm{v}_{\mathrm{y} 0} / \mathrm{c}=-0.009 \quad--\Psi, \mathrm{v}_{\mathrm{y} 0} / \mathrm{c}=-0.009
\end{aligned}
$$

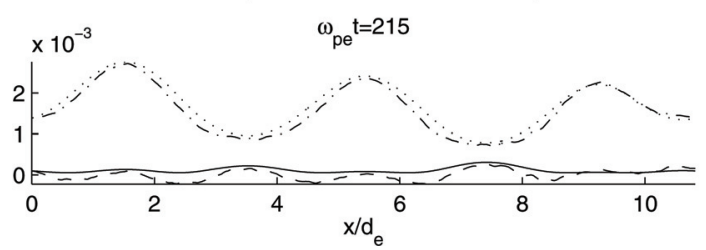

FIG. 7. (Color online) Logarithmic phase space (a) and potential fields (b) for particles with $-0.009 \leq v_{y 0} / c \leq 0.052$ at $\omega_{p e} t \approx 215$, the time of stream formation in Fig. 4(a), for the simulation with $L_{x} / d_{e}=10.81$. In panel (b), the potential fields with $(\Psi)$ and without $\left(\Psi^{\star}\right)$ the electrostatic contribution are depicted for the particles of the class with extreme velocities. 


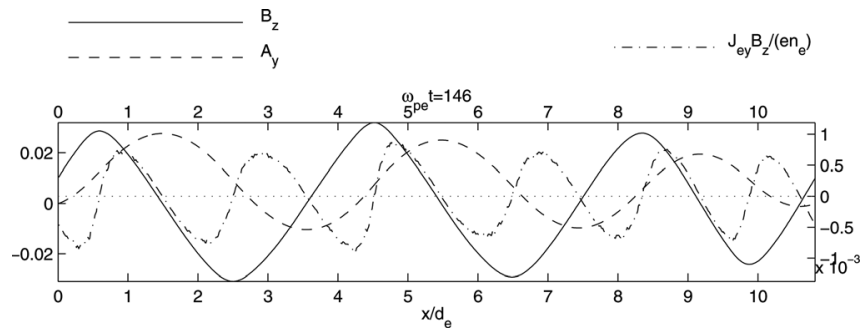

FIG. 8. Relevant components $B_{z}$ (straight line) and $A_{y}$ (dashed line) of the magnetic field and of the vector potential at saturation, $\omega_{p e} t \approx 146$, for the simulation with $L_{x} / d_{e}=10.81$. Instead of the electric field, $E_{x}, J_{e y} B_{z} /\left(e n_{e}\right)$ (dashed-dotted line, with ordinate on the right axis) is depicted because of the lower level of noise.

trapped. Instead, the electric field, which that is negative at $7.4 \lesssim x / d_{e} \lesssim 8.3$ and positive at $6.5 \lesssim x / d_{e} \lesssim 7.4$, accelerates the electrons away from the trapping positions. ${ }^{16}$ If the electrons reach $x / d_{e} \gtrsim 9.2$ and $x / d_{e} \lesssim 5.4$, they are accelerated to higher and lower $x / d_{e}$, respectively, both by the Lorentz force and by the electric field.

Given the mutual relations between $B_{z}, A_{y}$ and $E_{x}$, particles with low and positive $v_{y 0} / c$ originate streams at the $B_{z}$ zeros where the derivative of the field is negative. The stream profile follows the one of $-B_{z}$, because the majority of the particles in this class have positive perpendicular velocity.

The dynamics of particles with low and negative $v_{y 0} / c$ is similar. The streams form first in locations corresponding to the zeros of $B_{z}$ where the derivative of the field is positive, and they are then accelerated. The streams follow the profile of $B_{z}$, because the particles have negative $v_{y}$. The $E_{x}$ contribution is determinant in freeing the particles in this case also.

Thus, it is possible to trace back the streams in Fig. 4(a) to the class of particles which originated them: the streams at $x / d_{e} \approx 5.4$ and $x / d_{e} \approx 9.2$ are due to particles with an absolute value of the initial perpendicular velocity low and positive (see also Fig. 7), while the ones corresponding to the zeros of the magnetic field at $x / d_{e} \approx 3.5, x / d_{e} \approx 7.4$ and $x / d_{e} \approx 10.6$ are caused by particles with an absolute value of the initial perpendicular velocity low and negative. However, it is clear by comparing Figs. 4(a) and 7(a) that the particles with $-0.009 \leq v_{y 0} / c \leq 0.052$ do not account for all the streams at $x / d_{e} \approx 5.4$ and $x / d_{e} \approx 9.2$ : the "missing" particles, which form the streams at $6.5 \lesssim x / d_{e} \lesssim 7.5, x / d_{e} \gtrsim 9.5$ (forward propagating) and $6 \lesssim x / d_{e} \lesssim 7.5$ (backward propagating), are provided by the particles with lower $v_{y 0}$ from the next class, which experience a bigger "kick" from Lorentz force due to their higher $v_{y}$. Particles with even higher $v_{y 0}$ do not participate in the stream formation because for them the confining force, i.e. Lorentz force, is considerably larger than the freeing force due to the electric field.

Thus, it is possible to conclude that the streams originate from particles with low $v_{y 0} / c$ in correspondence to the $B_{z}$ zeros in the cases where the post-saturation evolution of the Weibel instability follows the first simulation evolution, where the dominant mode remains the same also after saturation. The streams are thin when compared to the bulk electron distribution, and the electron holes affect only the flanks of it. The fact that the longitudinal electric field $E_{x}$ has double wavenumber than $B_{z}$ is fundamental in freeing the par-

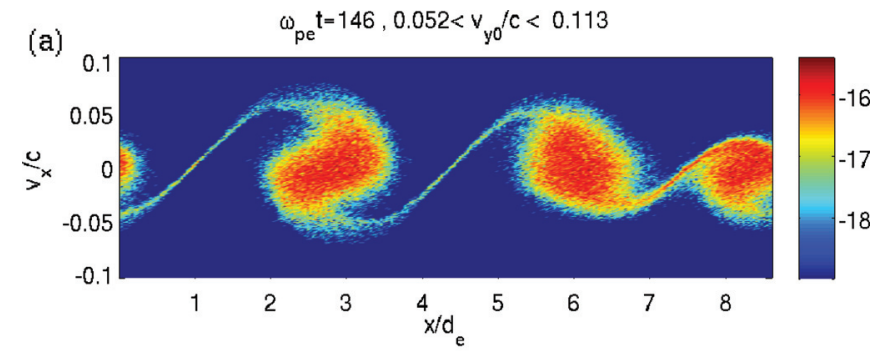

(b)

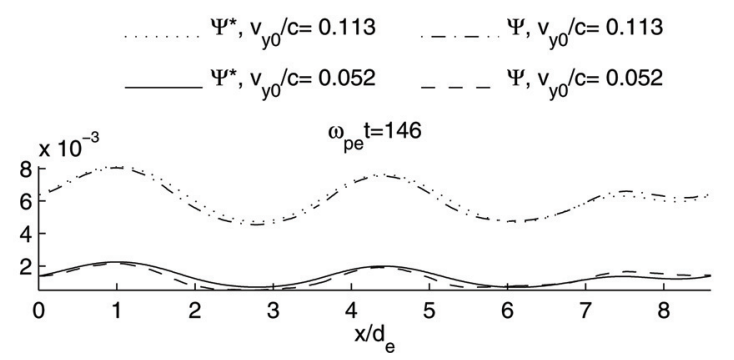

FIG. 9. (Color online) Logarithmic phase space (a) and potential fields (b) for particles with $0.052 \leq v_{y 0} / c \leq 0.113$ at $\omega_{p e} t \approx 146$, when saturation of the mode with $n=3$ occurs, for the simulation with $L_{x} / d_{e}=8.6$. In panel (b), the potential fields with $(\Psi)$ and without $\left(\Psi^{\star}\right)$ the electrostatic contribution are depicted for the particles of the class with extreme velocities.

ticles from their trapping positions, because $E_{x}$ tends to accelerate electrons away from them. This is proved by the fact that particles with higher perpendicular velocities, for which the Lorentz force is much stronger than the freeing force exerted by the electric field, do not participate in the stream formation. The role of $E_{x}$ is also confirmed by the fact that particle streams are not formed when the $2 n$ sinusoidal structure of $E_{x}$ is not generated (see the parametric study in Sec. VI).

Also the different post-saturation behavior of the simulation with $L_{x} / d_{e}=8.6$ can be understood in terms of the different trapping positions of particles. Fig. 9 shows the phase space (panel a) and the potential fields with $(\Psi)$ and without $\left(\Psi^{\star}\right)$ the electrostatic contribution for particles in the velocity class $0.052 \leq v_{y 0} / c \leq 0.113$ at saturation $\left(\omega_{p e} t \approx 146\right)$ for the simulation with $L_{x} / d_{e}=8.6$. The particles are trapped in three potential wells $\left(2 \lesssim x / d_{e} \lesssim 3.5,5.5 \lesssim x / d_{e} \lesssim 6.5\right.$ and $\left.x / d_{e} \gtrsim 7.5\right)$, which correspond to the $A_{y}$ minima because $v_{y 0} / c>0$. The electrostatic contribution does not change considerably the profile of the potential field because $\left|v_{y 0}\right| / c$ is high (see Eq. 10), and the third well is more shallow than the others because the mode of $B_{z}$ with $n=2$ has a growth rate comparable with the one dominant until now, $n=3$. The formation of the stream visible in Fig. 5(a) at $5.5 \lesssim x / d_{e} \lesssim 7.5$ is clear from Fig. 10, which shows the phase space plot (panel a) and the potential fields (panel b) for the same class of particles at $\omega_{p e} t \approx 185$, when streams are already visible in Fig. 5(a), and the dominant mode of $B_{z}$ is the one with $n=2$. The mode shift from $n=3$ to $n=2$ causes a modification in the profile of the potential fields: the third well is lifted and the particles previously trapped there are consequently accelerated by the $x$ component of the Lorentz force in the $-x$ direction $\left(B_{z}\right.$ is positive in that region of space). The stream in Fig. 5(a) at $x / d_{e} \gtrsim 8$ and $x / d_{e} \lesssim 1$ (due to the periodic condition on particles and fields) is mainly 
(a) $\omega_{p e} t=185,0.052<v_{y 0} / c<0.113$

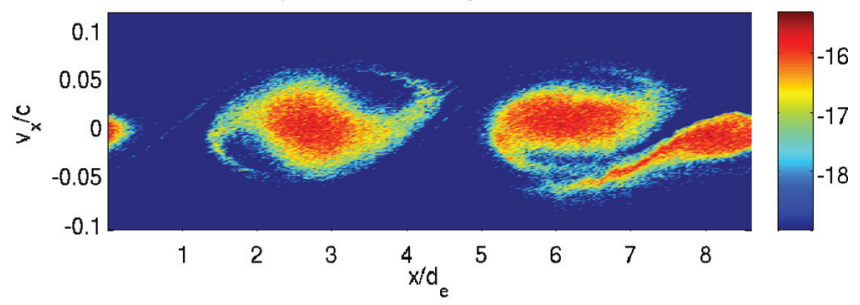

(b)

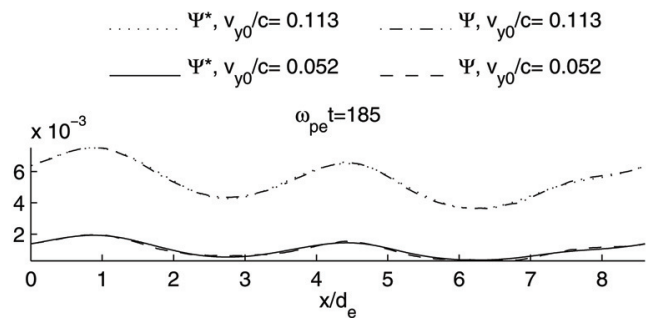

FIG. 10. (Color online) Logarithmic phase space (a) and potential fields (b) for particles with $0.052 \leq v_{y 0} \leq 0.113$ at $\omega_{p e} t \approx 185$, the time of stream formation in Fig. 5, for the simulation with $L_{x} / d_{e}=8.6$. In panel (b), the potential fields with $(\Psi)$ and without $\left(\Psi^{\star}\right)$ the electrostatic contribution are depicted for the particles of the class with extreme velocities.

formed by particles with $-0.131 \leq v_{y 0} / c \leq-0.070$ (high negative $v_{y 0} / c$ ) similarly affected by the mode shift.

The fact that the mode shift originates a strong secondary two stream instability does not imply that the weaker two stream instability from particles with $v_{y 0} / c \approx 0$ is prevented from developing. In fact, it is clear from Fig. 5(b) that the thinner streams at $4.6 \lesssim x / d_{e} \lesssim 6.5$ and $1.5 \lesssim x / d_{e} \lesssim 2.5$ originate from particles with low initial perpendicular velocity. The mechanism is comparable to the one described for the first simulation.

\section{PARAMETRIC STUDY}

Because the velocity anisotropy here used, $v_{t h, \perp} /$ $v_{t h . \|}=6$, is rather high when compared to the ones usually employed in similar studies, a parametric study is carried out to determine if and at which anisotropy values the described evolutions change. Simulations with $v_{t h, \perp} / v_{t h, \|}=4$ and $v_{t h, \perp} / v_{t h, \|}=2$ have been completed for the case without and with mode shift. In the case with $v_{t h, \perp} / v_{t h, \|}=4$, a box length of $L_{x} / d_{e}=13.85$ is chosen to have the mode with higher wavenumber from the linear theory, $k d_{e}=1.36$, contained in the simulation box exactly three times. Instead, the box is chosen with $L_{x} / d_{e}=10.62$ to enable mode shift from $n=3$ to $n=2$. In both cases, the time step $\omega_{p e} \Delta t$ is increased to $\omega_{p e} \Delta t=0.0048$ because of the decreased growth rate of the instability at a lower anisotropy.

Instead, in the case with $v_{t h, \perp} / v_{t h, \|}=2$, where the wavenumber with maximum growth rate is $k d_{e}=0.8$, the box is selected with $L_{x} / d_{e}=23.55$ and $L_{x} / d_{e}=18.05$ to prevent and allow mode shift. The time step is now increased to $\omega_{p e} \Delta t=0.0082$.

Figure 11 shows the time space plot of the longitudinal electric field (panel a) and the logarithmic phase space plot at $\omega_{p e} t \approx 410$ (panel b) for the simulation with $v_{t h, \perp} /$ $v_{t h, \|}=4$ and $L_{x} / d_{e}=13.85$, where the mode shift does not occur. Figures 11(a) and 11(b) are qualitatively similar to the
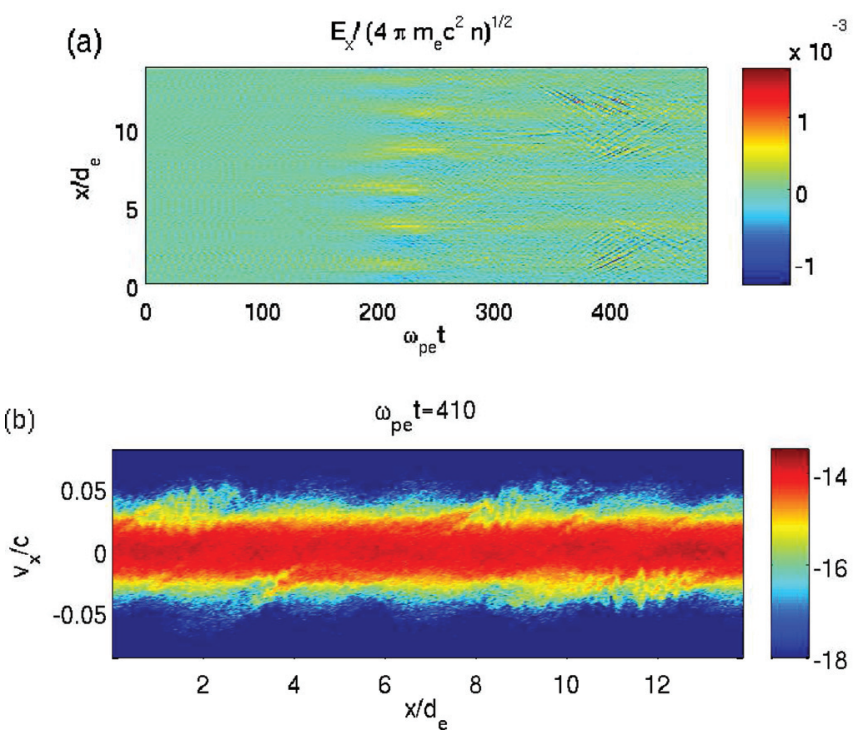

FIG. 11. (Color online) Time space plot of $E_{x}$ (panel a) and phase space plots at $\omega_{p e} t \approx 410$ (panel b) for the simulation with $v_{t h, \perp} / v_{t h, \|}=4, L_{x} /$ $d_{e}=13.85$ and no mode shift.

same quantities for $v_{t h, \perp} / v_{t h, \|}=6$ and no mode shift, as shown in Figs. 2(b) and 4(b). The longitudinal electric field exhibits a sinusoidal structure with a double wavenumber with respect to one of the magnetic field. Moreover, traces appearing at a time which corresponds roughly to twice the saturation time $\left(\omega_{\text {pe }} t_{\text {saturation }} \approx 250\right)$ testify the onset of a secondary two stream instability. This is confirmed by the phase space plot at a time where traces are already visible in Fig. 11(a) also. Moreover, the analysis of the different classes of particles reveals that the mechanism of stream formation is the same: particles with low $v_{y 0} / c$ are first freed from their potential wells by the electric field and then accelerated into streams by both the electric field and the Lorentz force.

In addition, the role of $E_{x}$ is confirmed by Fig. 12, where the space time plot of the longitudinal electric field (panel a)

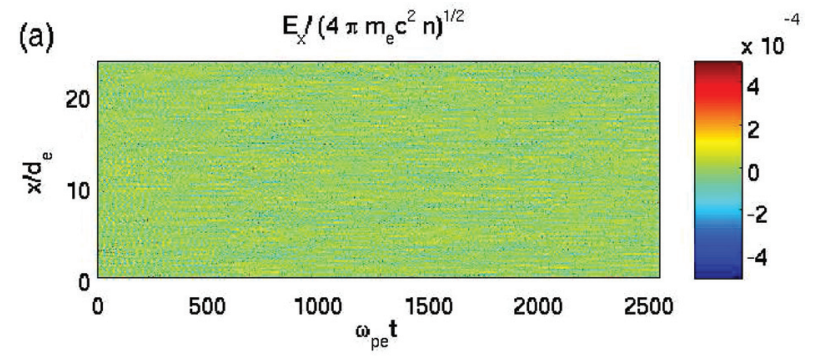

(b)

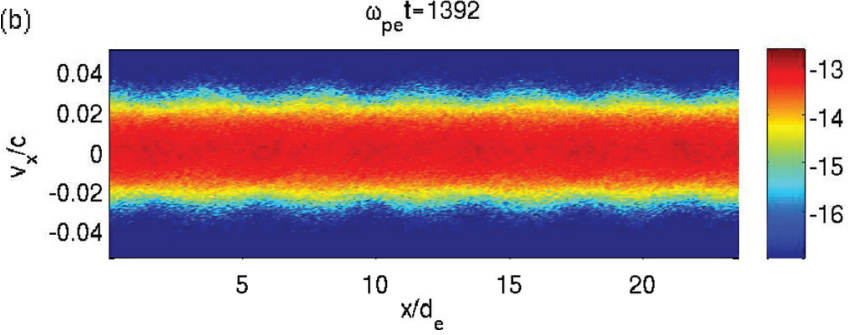

FIG. 12. (Color online) Time space plot of $E_{x}$ (panel a) and phase space plots at $\omega_{p e} t \approx 1392$ (panel b) for the simulation with $v_{t h, \perp} / v_{t h . \|}=2, L_{x} /$ $d_{e}=23.55$ and no mode shift. 
(a)
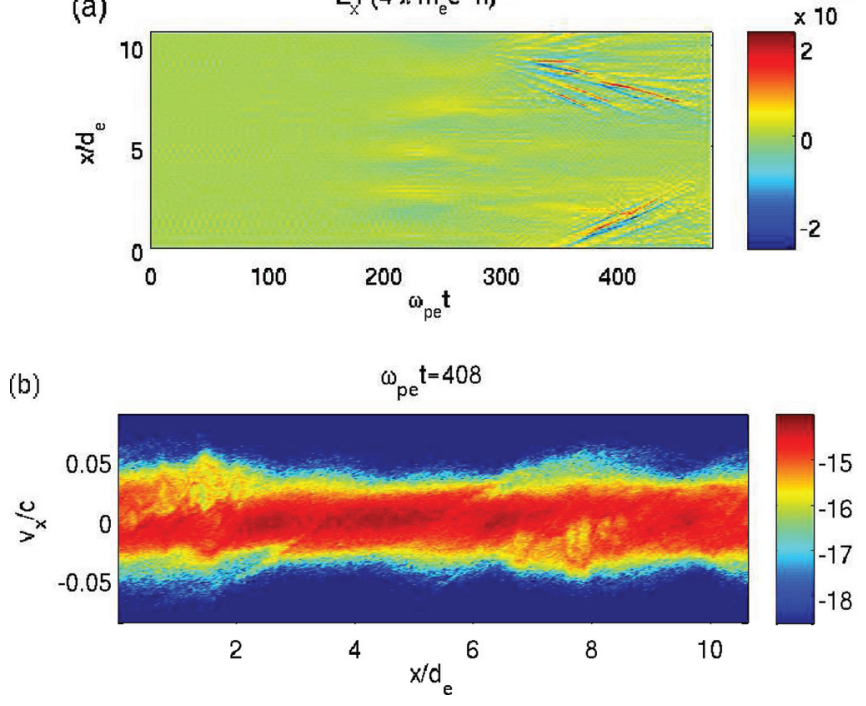

FIG. 13. (Color online) Time space plot of $E_{x}$ (panel a) and phase space plots at $\omega_{p e} t \approx 408$ (panel b) for the simulation with $v_{t h, \perp} / v_{t h, \|}=4, L_{x} /$ $d_{e}=10.62$ and mode shift.

and the phase space plot at twice the saturation time (here, $\omega_{\text {pe }} t_{\text {saturation }} \approx 680$ ) are shown for the simulation with $v_{t h, \perp} / v_{t h, \|}=2$ and no mode shift, $L_{x} / d_{e}=23.55$. The particular time of the phase space plot has been chosen because the simulations with higher anisotropies and no mode shift previously shown have already developed the secondary two stream instability at twice the saturation time (see Figs. 2b and 11a).

The longitudinal electric field is not compressed into the characteristic sinusoidal structure in the case of such an anisotropy. Phase space plots of the separate classes of particles show, consistently, that all electrons are trapped in three and not six potential wells. The clusters of particles are wider in the $x$ direction than in the previous cases, because the wells are shallower. Without the sinusoidal electric field accelerating electrons out of the wells, streams, and consequently the secondary two stream instability, are not originated, as it is clear from Figs. 12(a) and 12(b).

The time space plot of $E_{x}$ and the phase space plot at $\omega_{p e} t \approx 408$ are shown in Fig. 13 for the simulation with $v_{t h, \perp} / v_{t h, \|}=4, L_{x} / d_{e}=10.62$ and mode shift. Both Figs. 13(a) and 13(b) show a behavior which is qualitatively similar to the one of the simulation with $v_{t h, \perp} / v_{t h, \|}=6$ and mode shift: traces of a strong secondary two stream instability are superimposed to the sinusoidal structure of $E_{x}$. Consistently with the evolution presented in Sec. V, the strong traces originate from a single location in space corresponding to the position of the potential well lifted by mode shift and are caused by the evolution of the secondary two stream instability into electron holes. This is evident in Fig. 13(b). The weaker streams originate from particles with lower $\left|v_{y 0}\right| / c$ in correspondence with the zeros of $B_{z}$ at $x / d_{e} \approx 3.5$ and $x / d_{e} \approx 5.5$.

Instead, the time space plot of $E_{x}$ and the phase space plot for electrons at $\omega_{\text {pe }} t \approx 2296$ are shown in Fig. 14 for the simulation with $v_{t h, \perp} / v_{t h, \|}=2, L_{x} / d_{e}=18.05$ and mode shift. The spectral analysis shows that the mode with $n=3$,
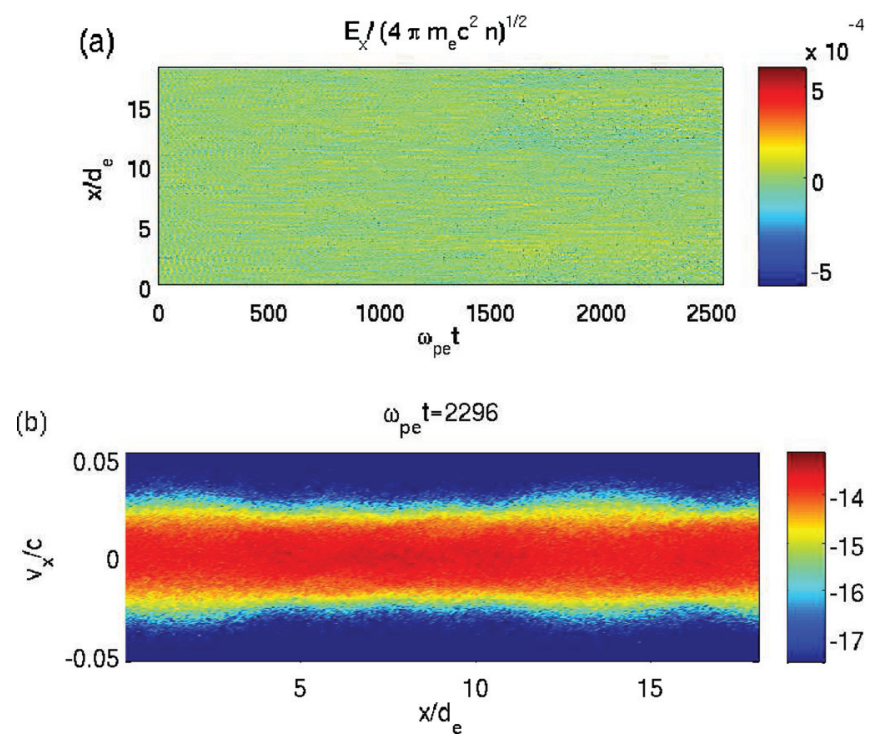

FIG. 14. (Color online) Time space plot of $E_{x}$ (panel a) and phase space plots at $\omega_{p e} t \approx 2296$ (panel b) for the simulation with $v_{t h, \perp} / v_{t h, \|}=2, L_{x} /$ $d_{e}=18.05$ and mode shift.

$k d_{e}=1.04$ saturates at $\omega_{p e} t \approx 705$ and the mode with $n=2$, $k d_{e}=0.7$, becomes dominant at $\omega_{p e} t \approx 1650$. In fact, the analysis of the evolution of the different classes of particles reveals that electrons are trapped coherently with the potential wells positions and, therefore, the dominant wavenumber, as it happened in the simulations with higher anisotropies and mode shift. However, the Lorentz force acceleration that the particles experience is not strong enough to accelerate them in streams, as proved by the phase space plot in Fig. 14(b), which refers to a time when the wavenumber with $n=2$ is already dominant. Note the rounded halo around the bulk electron distribution which reflects the particle trapping in two wells according to their perpendicular velocity: particles with high negative $\left|v_{y 0}\right| / c$ are trapped at $x / d_{e} \lesssim 4.5, x / d_{e} \gtrsim 16$ (this is a unique well due to the periodic conditions) and $6 \lesssim x / d_{e} \lesssim 12$, particles with high positive $\left|v_{y 0}\right| / c$ at $2.5 \lesssim x / d_{e} \lesssim 9$ and $10.5 \lesssim$ $x / d_{e} \lesssim 17$.

The secondary two stream instability development appears to be impaired by anisotropies $v_{t h, \perp} / v_{t h, \|} \leq 2$ also in the case with a mode shift. However, in this case, the fact that the longitudinal electric field is not compressed into a sinusoidal structure does not seem to play any role. Instead, the absence of streams is related to the weaker magnetic field $B_{z}$ due to the lower initial anisotropy.

\section{CONCLUSIONS}

In this paper, the electrostatic activity arising in 1D 3V simulations after the saturation of the classical Weibel instability has been analyzed. Two 1D 3V particle in cell simulations have been presented. In the first case, the box length has been chosen in order to allow the wavenumber with maximum growth rate from the linear theory to resonate in it an entire number of times. In the second simulation, the box length is chosen so that the dominant mode of the magnetic field shifts from a higher to a lower wavenumber during the 
simulation. This is regarded as a way to reproduce, in a controlled way, the progressive stabilization from higher to lower wavenumbers, which is expected from the Weibel instability as the electron distribution function gets more and more isotropic ${ }^{23}$ and, therefore, as a more realistic evolution scenario for the instability.

Four main results are presented in this paper.

First, the electrostatic activity has been identified as a secondary two stream instability. Its evolution strongly depends on the possibility of mode shift (shifting of the dominant wavenumber of the magnetic field from higher to lower values) during the simulation. It has been shown that if a mode shift is not allowed, the streams are thin and generated in multiple positions, and the electron holes affect just the flanks of the phase space distributions. Instead, if a mode shift is allowed the streams depart from just one position and their evolution generates electron holes of size comparable with the bulk phase space distribution and stronger peaks in the electric field.

Second, it has been proved that the Hamiltonian description of particle motion in a 1D setting is a valuable tool to pinpoint the origin and evolution of the streams. The effective potential that the particles experience as a function of their initial perpendicular velocity has been calculated. Its minima match with the trapping positions of the different classes of particles, sorted according to their initial perpendicular velocity. The role of the electric field in determining the trapping positions of low $v_{y 0} / c$ particles, neglected in Morse and Nielson, ${ }^{21}$ has been highlighted.

Third, the different origins of the streams in the evolution scenarios without and with a mode shift have been characterized. Without a mode shift, the streams originate from particles with low absolute value of the initial perpendicular velocities. These particles are freed in a limited number from their trapping positions in correspondence to the magnetic field zeros. It has been highlighted that the sinusoidal profile of the electric field is crucial in accelerating the electrons away from their trapping positions. Instead, with a mode shift, the thicker streams have been shown to be due to particles with an initial high absolute value of the perpendicular velocity, which are freed from the potential well lifted by the mode shift. This explains why, in this case, the streams are thicker (more particles are involved in the stream formation than in the previous case) and originated from the same location of space.

Fourth, a parametric study has shown that the effects described here for a velocity anisotropy of $v_{t h, \perp} / v_{t h, \|}=6$ are not produced with anisotropies as low as $v_{t h, \perp} / v_{t h, \|}=2$. In the case without a mode shift, the absence of streams has been related to the fact that the sinusoidal structure in the longitudinal electric field is not generated, thus preventing the electron extraction for the potential field wells. Instead, in the case with mode shift, the crucial factor which prevents the formation of streams is the fact that Lorentz force is not strong enough to accelerate the particles freed by the potential well lifting into streams with high enough $v_{y} / c$.

\section{ACKNOWLEDGMENTS}

This work was supported by the NASA MMS Mission Theory Support, by the Onderzoekfonds KULeuven (Research Fund KULeuven), and by the European Commission's Seventh Framework Programme (FP7/2007-2013) under the Grant Agreement No. 218816 (SOTERIA project, www.soteria-space.eu). The simulations were conducted on the resources of the NASA Advanced Supercomputing Division (NAS), of the The NASA Center for Computational Sciences Division (NCCS), and of the Vlaams Supercomputer Centrum (VSC) at the Katholieke Universiteit Leuven.

${ }^{1}$ E. S. Weibel, Phys. Rev. Lett. 2, 83 (1959).

${ }^{2}$ B. D. Fried, Phys. Fluids 2, 337 (1959).

${ }^{3}$ G. Kalman, C. Montes, and D. Quemada, Phys. Fluids 11, 1797 (1968).

${ }^{4}$ N. Albright, Phys. Fluids 13, 1021 (1970).

${ }^{5}$ M. Lazar, R. Schlickeiser, R. Wielebinski, and S. Poedts, Astrophys. J. 693, 1133 (2009).

${ }^{6}$ A. Bret, Astrophys. J. 699, 990 (2009).

${ }^{7}$ T. N. Kato, Phys. Plasmas 12, 080705 (2005).

${ }^{8}$ F. Califano, F. Pegoraro, S. V. Bulanov, and A. Mangeney, Phys. Rev. E 57, 7048 (1998).

${ }^{9}$ M. Medvedev and A. Loeb, Astrophys. J. 526, 697 (1999).

${ }^{10}$ F. Califano, T. Cecchi, and C. Chiuderi, Phys. Plasmas 9, 451 (2002).

${ }^{11}$ R. Schlickeiser and P. Shukla, Astrophys. J. 599, L57 (2003).

${ }^{12}$ R. A. Fonseca, L. O. Silva, J. W. Tonge, W. B. Mori, and J. M. Dawson, Phys. Plasmas 10, 1979 (2003).

${ }^{13}$ C. Briand, Nonlinear Processes Geophys. 16, 319 (2009).

${ }^{14}$ H. Matsumoto, H. Kojima, T. Miyatake, Y. Omura, M. Okada, I. Nagano, and M. Tsutsui, Geophys. Res. Lett. 21, 2915, doi:10.1029/94GL01284 (1994).

${ }^{15}$ A. A. Galeev and R. Z. Sagdeev, Basic Plasma Physics: Selected Chapters, Handbook of Plasma Physics Vol. 1, edited by A. A. Galeev and R. N. Sudan, (North-Holland Publishing Company, Amsterdam, 1984).

${ }^{16}$ A. Stockem, M. E. Dieckmann, and R. Schlickeiser, Plasma Phys. Controlled Fusion 51, 075014 (2009).

${ }^{17}$ L. Palodhi, F. Califano, and F. Pegoraro, Plasma Phys. Controlled Fusion 51, 125006 (2009).

${ }^{18}$ H. Schamel, Phys. Rev. Lett. 48, 481 (1982).

${ }^{19}$ B. Eliasson and P. Shukla, Nonlinear Processes Geophys. 12, 269 (2005).

${ }^{20} \mathrm{G}$. Schmidt, Physics of High Temperature Plasmas (Academic, New York, 1979).

${ }^{21}$ R. L. Morse and C. W. Nielson, Phys. Fluids 14, 830 (1971).

${ }^{22}$ T.-Y. B. Yang, J. Arons, and A. B. Langdon, Phys. Plasmas 1, 3059 (1994).

${ }^{23}$ R. C. Davidson, D. A. Hammer, I. Haber, and C. E. Wagner, Phys. Fluids 15, 317 (1972).

${ }^{24}$ B. D. Fried and S. D. Conte, in The Plasma Dispersion Function, edited by B. D. Fried and S. D. Conte (Academic, New York, 1961).

${ }^{25}$ S. Markidis, G. Lapenta, and Rizwan-uddin, Math. Comput. Simul. 80, 1509 (2010). 